\title{
Critical analysis and comment (News Perfor-
}

\section{mance)}

\section{AUTHOR}

Edda Humprecht

\section{KEYWORDS}

news coverage, investigative journalism, watchdog reporting, criticism

FIELD OF APPLICATION/THEORETICAL FOUNDATION Analyses of critical analysis and comment are theoretically linked to the news performance and the watchdog function of the media (Donsbach, 1995; McQuail, 1992). This construct is related to the normative expectation that the news media should critically analyze and comment on cases of abuse of power, incompetence, failures and grievances in government institutions, nonprofit organizations, or the private sector (Downie \& Schudson, 2009).

\section{REFERENCES/COMBINATION WITH OTHER} METHODS OF DATA COLLECTION

The analysis of critical reporting and comment is complex and requires an understanding of the context and the references made by the journalist. Furthermore, it is empirically demanding to distinguish between critical reporting in the sense of the watchdog function and criticism in the sense of negativity or sensationalism (Humprecht, 2016). Due to this complexity, automated approaches have hardly been employed so far.

\section{EXAMPLE STUDIES:}

Benson (2010); Humprecht (2016)

\section{REFERENCES}

Benson, R. (2010). What Makes for a Critical

Press? A Case Study of French and U.S.

Immigration News Coverage. The Interna-

tional Journal of Press/Politics, 15(1), 3-24. https://doi.org/10.1177/1940161209349346

Donsbach, W. (1995). Lapdogs, Watchdogs and Junkyard Dogs. Media Studies Journal, Fall 1995, 17-30.

Downie, L., \& Schudson, M. (2009). The Reconstruction of American Journalism.

Humprecht, E. (2016). Shaping Online News Performance. In Palgrave Macmillan. Palgrave Macmillan UK. https:// doi.org/10.1007/978-1-137-56668-3

McQuail, D. (1992). Media Performance: Mass Communication and the Public Interest. Sage Publications. 


\begin{tabular}{|c|c|c|c|c|}
\hline Author(s) & Sample & Unity of Analysis & Values & Reliability \\
\hline $\begin{array}{l}\text { Benson } \\
(2010)\end{array}$ & $\begin{array}{l}\text { Content type: im- } \\
\text { migration news } \\
\text { coverage (all } \\
\text { articles focused } \\
\text { on broad immi- } \\
\text { gration trends, } \\
\text { policy making } \\
\text { and politics, or } \\
\text { individual im- } \\
\text { migrants) } \\
\text { Outlet/ country: } \\
\text { 14 newspapers } \\
\text { from two count- } \\
\text { ries (FR, US) } \\
\text { Sampling peri- } \\
\text { od: } 1991 / 1994 ; \\
\text { 2002/2004; 2006) } \\
\text { Sample size: N= } \\
\text { 1088 }\end{array}$ & $\begin{array}{l}\text { Unit of analysis: cri- } \\
\text { tical statements in } \\
\text { news articles (from } \\
\text { sources/ journa- } \\
\text { lists) } \\
\text { Critical statements } \\
\text { are classified accor- } \\
\text { ding to their target, } \\
\text { substantive focus, } \\
\text { and sources } \\
\text { Target (govern- } \\
\text { ment; dominant left } \\
\text { parties; dominant } \\
\text { right parties; minor } \\
\text { political parties; } \\
\text { civil society organi- } \\
\text { zations; business; } \\
\text { foreign or internati- } \\
\text { onal organizations) } \\
\text { Focus (administrati- } \\
\text { ve, character, truth, } \\
\text { ideology, policy, } \\
\text { and strategy) }\end{array}$ & $\begin{array}{l}\text { Administrative criticism } \\
\text { (failure (e.g., corruption, } \\
\text { incompetence, mismanage- } \\
\text { ment) } \\
\text { Truth criticism (e.g., evi- } \\
\text { dence to demonstrate the } \\
\text { falsity of claims) } \\
\text { Character criticisms (e.g., } \\
\text { attacks on personal charac- } \\
\text { teristics of powerful indivi- } \\
\text { duals in public life) } \\
\text { Policy criticism (e.g., logical } \\
\text { coherence, feasibility, em- } \\
\text { pirical justification, eviden- } \\
\text { ce supporting any pro- po- } \\
\text { sed policy) } \\
\text { Ideology criticism (e.g., cri- } \\
\text { ticisms of fascism, racism, } \\
\text { sexism, other worldviews) } \\
\text { Strategy criticisms (nega- } \\
\text { tive assessments of effecti- } \\
\text { veness of a particular idea/ } \\
\text { action; normative criticisms } \\
\text { of political strategies) }\end{array}$ & $\begin{array}{l}\text { Holsti } \\
M=0.85\end{array}$ \\
\hline $\begin{array}{l}\text { Hum- } \\
\text { precht } \\
\text { (2016) }\end{array}$ & $\begin{array}{l}\text { Content type: Poli- } \\
\text { tical routine-peri- } \\
\text { od online news } \\
\text { Outlet/ country: } \\
48 \text { online news } \\
\text { outlets from six } \\
\text { countries (CH, } \\
\text { DE, FR, IT, UK, } \\
\text { US) } \\
\text { Sampling period: } \\
\text { June - July } 2012 \\
\text { Sample size: } \mathrm{N}= \\
1660\end{array}$ & $\begin{array}{l}\text { Unit of analysis: } \\
\text { Political news items } \\
\text { (make reference to } \\
\text { a political actor, e.g. } \\
\text { politician, party, } \\
\text { institution in head- } \\
\text { line, sub-headline, } \\
\text { in first paragraph } \\
\text { or in an accompa- } \\
\text { nying visual) }\end{array}$ & $\begin{array}{l}\text { Story shows critical per- } \\
\text { spective towards authori- } \\
\text { ties/power holders } \\
\text { Story raises probing ques- } \\
\text { tions at actors responsible } \\
\text { for a problem } \\
\text { Story discovers new, previ- } \\
\text { ously unknown information } \\
\text { about a problem of social/ } \\
\text { political relevance; story } \\
\text { may unveil a 'scandal' }\end{array}$ & $\begin{array}{l}\text { Cohen's } \\
\text { kappa: } \\
\text { critical } \\
\text { perspective } \\
=0.74 \\
\text { probing } \\
\text { questions = } \\
0.67 \\
\text { unveiling } \\
\text { scandals = } \\
0.81\end{array}$ \\
\hline
\end{tabular}

